\title{
Complement receptor 3 forms a compact high affinity complex with
}

\section{iC3b}

Rasmus K. Jensen ${ }^{1}$, Goran Bajic ${ }^{2,3}$, Mehmet Sen ${ }^{4}$, Timothy A. Springer ${ }^{5,6}$, Thomas VorupJensen ${ }^{7}$, Gregers R. Andersen ${ }^{1 *}$

${ }^{1}$ Department of Molecular Biology and Genetics, Aarhus University, Denmark

${ }^{2}$ Laboratory of Molecular Medicine, Boston Children's Hospital, Harvard Medical School, Boston, MA 02115, USA

${ }^{3}$ Department of Pediatrics, Harvard Medical School, Boston, MA, 02115, USA

${ }^{4}$ Department of Biology and Biochemistry, University of Houston, Houston, TX, 77204, USA

${ }^{5}$ Department of Biological Chemistry and Molecular Pharmacology, Harvard Medical School, Boston, MA 02115, USA

${ }^{6}$ Program in Cellular and Molecular Medicine, Boston Children's Hospital, Boston, MA, USA

${ }^{7}$ Department of Biomedicine, Aarhus University, Denmark

* Corresponding author. Email: gra@mbg.au.dk 


\begin{abstract}
Complement receptor 3 (CR3, also known as Mac-1, integrin $\alpha_{M} \beta_{2}$, or CD11b/CD18) is expressed on a subset of myeloid and certain activated lymphoid cells. CR3 is essential for the phagocytosis of complement-opsonized particles such as pathogens and apoptotic or necrotic cells opsonized with the complement fragment iC $3 \mathrm{~b}$ and to a lesser extent $\mathrm{C} 3 \mathrm{dg}$. While the interaction between the iC3b thioester domain and the ligand binding CR3 $\alpha_{M}$ I-domain is structurally and functionally well characterized, the nature of additional CR3-iC3b interactions required for phagocytosis of complement opsonized objects remain obscure. Here we analyzed the interaction between ic3b and the $150 \mathrm{kDa}$ headpiece fragment of the CR3 ectodomain. Surface plasmon resonance experiments demonstrated a $30 \mathrm{nM}$ affinity of $\mathrm{CR} 3$ for ic $3 \mathrm{~b}$ compared to $515 \mathrm{nM}$ for the iC3b thioester domain. Small angle $\mathrm{x}$-ray scattering analysis revealed that iC $3 \mathrm{~b}$ adopts an extended but preferred conformation in solution. Upon interaction with $\mathrm{CR} 3$, iC3b rearranges to form a compact receptor-ligand complex. Overall, the data suggest that the iC3b-CR3 interaction is of high affinity and relies on minor contacts formed between $\mathrm{CR} 3$ and regions outside the iC $3 \mathrm{~b}$ thioester domain. Our results rationalize the more efficient phagocytosis elicited by iC3b than by C3dg and pave the way for development of specific therapeutics for treatment of inflammatory and neurodegenerative diseases that do not interfere with recognition of non-complement CR3 ligands.
\end{abstract}




\section{Introduction}

The complement system is a central part of vertebrate innate immunity. It connects to other branches of the immune system, including adaptive immunity through its functions especially in stimulation of antibody formation. Complement is a tightly regulated proteolytic cascade, which upon activation leads to cleavage of the $186 \mathrm{kDa}$ complement component 3 (C3) into an anaphylatoxin $\mathrm{C} 3 \mathrm{a}$ and the opsonin C3b (Fig. 1A). C3b is deposited on the surface of the complement activator through covalent bond formation when an activator nucleophile reacts with an exposed thioester (TE) present in the TE domain of nascent C3b. Host cells present glycans that attract the fluid phase regulator factor $\mathrm{H}(\mathrm{FH})$, and also express complement regulators membrane cofactor protein (MCP/CD46) and CR1/CD35. These regulators bind specifically to C3b and enable its degradation by the protease factor I (FI). As a result, $\mathrm{C} 3 \mathrm{~b}$ is quickly converted to iC3b (1), which acts as a powerful opsonin, as it is recognized by complement receptor 2 (CR2) as well as the two integrin receptors CR3 (CD11b/CD18 or integrin $\left.\alpha_{M} \beta_{2}\right)$ and CR4 (p150,95, CD11c/CD18 or integrin $\alpha \times \beta_{2}$ ). Whereas C3b has a well-defined conformation, double cleavage by FI of C3b in one of two connections to its CUB domain leads to a flexible attachment of the thioester domain to the C3c moiety (Fig. 1A) (2-4). Hydrogen deuterium exchange suggested that in iC3b, the remnants of the degraded CUB domain become surface exposed and disordered (5).

Recognition of iC3b by CR3 leads to several physiological responses dependent on the cell type and activation state of the CR3-expressing cell, including phagocytosis of dying host cells or pathogens $(6,7)$. CR3, consisting of the non-covalently associated integrin $\alpha_{M}$ and $\beta_{2}$ subunits, is highly expressed on the plasma membrane of myeloid cells including macrophages, monocytes, dendritic cells and neutrophil granulocytes and is upregulated from storage granules upon stimulation. Certain lymphoid leukocytes such as natural killer cells and activated $\mathrm{T}$ cells also express CR3, and expression is further inducible in other leukocytes $(6,7)$. CR3 is also highly expressed in microglia, the mononuclear phagocytes of the central nervous system (CNS), where CR3-mediated phagocytosis of iC3b opsonized presynaptic termini of neurons is important for neural development and homeostasis (8-11). In vivo studies leave no doubt about the importance 
of CR3-supported mechanisms, both as a protective agent against infection (12) and as an aggravating factor in diseases with a poorly regulated inflammatory response, for instance, as observed in animal models of multiple sclerosis and Alzheimer's disease $(12,13)$. CR3 also plays a key role in complement stimulation of the adaptive immune system. Immune complexes containing complement-opsonized antigens drain with the afferent lymphatics into the subcapsular sinus where complement-opsonized antigens are taken up by subcapsular sinus macrophages via CR3 and carried across the subcapsular sinus floor. Next, the antigen is handed off to non-cognate B cells via complement receptor 2 which transport it into the follicle (14).

CR3, similar to other integrins, adopts at least three distinct conformations in the cell membrane, which control the activity of the protein. These conformations are the bent-closed and extendedclosed conformations with low affinity for ligands and the extended-open conformation with high affinity (15) (Fig. 1B). The conformation of integrins is regulated through inside-out signaling, where stimuli received by the cell through other receptors are signaled to the integrin, and outside-in signaling, where a ligand is recognized by the integrinThis signaling, together with the signal of tensile force relayed through the integrin, together stabilize the high affinity conformation (16-18).

In the CR3 $\alpha_{M}$ chain, the I-domain ( $\left.\alpha_{M} l\right)$ contains the primary ligand binding site, with a metal iondependent binding site (MIDAS) at its center. The I-domain binds a plethora of ligands including iC3b, ICAM-1, RAGE, platelet factor 4, mindin, platelet glycoprotein lb, sialylated FcyRIIA, CD40L, LL-37, LRP1, fibrinogen, and the LukAB cytotoxin (19-28). Many of these interactions seem at least in part to rely on the ability of the $\alpha_{M} l$ to weakly bind glutamate side chains, which translates into avid interactions from multivalent binding of membrane-tethered CR3 (29). By contrast, C3 fragments present distinct high-affinity interactions. We previously established that the major binding site for the CR3 $\mathrm{a}_{\mathrm{M}}$ is located in the TE domain of iC3b and this interaction is characterized by a dissociation constant $\left(K_{D}\right)$ of $600 \mathrm{nM}(30)$. However, prior studies suggested that the full-length receptor has a higher affinity for ic3b $(31,32)$, and other studies $(33-36)$ proposed one or more additional recognition sites between iC3b and CR3. A recent structural analysis by negative stain 
electron microscopy (nsEM) of the iC3b-CR3 headpiece complex suggested the proximity of regions in iC3b close to the $\mathrm{C} 345 \mathrm{c}$ domain and the $\beta$-propeller/ $\beta$ I-like domain portion of the CR3 headpiece. However, a three dimensional reconstruction was not obtained and the authors suggested that the tendency of the MG ring with its C345c domain in iC $3 b$ and the tendency of the CR3 headpiece to lay flat on EM grids may have broken a 3-dimensional interaction in the vicinity of the domains that were seen to lie close on grids (2).

To investigate whether the iC3b-CR3 complex is an ordered complex with a specific conformation or a flexible ensemble of conformations due to ic $3 b$ flexibility, we analyzed the complex between iC3b and the CR3 headpiece in solution through multiple biochemical and biophysical techniques. We now show that the CR3-iC3b interaction is characterized by a 17 -fold higher affinity than the minimal complex between the iC3b TE domain and the CR3 aM I-domain (30). SAXS rigid body analysis suggests that the iC3b undergoes a significant conformational change upon CR3 recognition allowing $\mathrm{CR} 3$ to interact with regions outside the iC3b thioester domain.

\section{Results}

\section{The CR3 headpiece forms a high affinity complex with iC3b}

The CR3 headpiece was purified from HEK293S $\mathrm{GnTl}^{-}$cells stably transfected with plasmids encoding the $\alpha_{\mathrm{M}}$ - and $\beta_{2}$-chains of the $140 \mathrm{kDa}$ CR3 headpiece fragment (dashed outline, Fig. 1B). We investigated the oligomerization state of our CR3 headpiece using analytical SEC in $\mathrm{Mn}^{2+}, \mathrm{Mg}^{2+}$, and $\mathrm{Ni}^{2+}$-containing buffers (Fig. S1A). At low concentrations, the CR3 headpiece in both $\mathrm{Mg}^{2+}$ and $\mathrm{Ni}^{2+}$ mainly eluted as a monomer, but a small dimer fraction was also present. Conversely, in $\mathrm{Mn}^{2+}$ CR3 eluted as a dimer in agreement with prior findings that the CR3 headpiece is prone to dimerization in a divalent metal-ion dependent fashion (2). Concentration-dependent dimerization was further confirmed by SAXS (Fig. S1B-E). To verify that the recombinant CR3 headpiece is able to form a stable complex with iC3b, we assessed the stability of the complex by SEC. The CR3:iC3b complex eluted earlier than either of the two individual proteins, in a monodisperse peak (Fig. 1C). 
We used surface plasmon resonance (SPR) to measure the affinity and the kinetics of the CR3 headpiece-ic3b interaction. We coupled C3b to biotin through its free thioester cysteine side chain and subsequently converted $\mathrm{C} 3 \mathrm{~b}$ to ic3b by $\mathrm{FI}$ cleavage in the presence of $\mathrm{FH}$ as previously described (37). The biotinylated ic3b was then bound to a streptavidin-coated SPR chip surface. The CR3 headpiece bound iC3b in a $\mathrm{Mg}^{2+}$ containing buffer with a dissociation constant $\mathrm{K}_{\mathrm{D}}=30$ nM when fitted to a 1:1 interaction model (Fig. 1D+F and Table S1). In a $\mathrm{Mn}^{2+}$ containing buffer, CR3 bound to iC3b with a significantly lower kon (Fig. 1E). The dissociation rate was however 30fold lower than with $\mathrm{Mg}^{2+}$, and the CR3 affinity for iC3b in the presence of $\mathrm{Mn}^{2+}$ was therefore approximately 5-fold higher with an apparent dissociation constant $K_{D}$ of 6.2 nM (Fig. 1E-F and Table S1).

\section{Additional iC3b binding sites are present in CR3}

The affinity of the CR3 headpiece binding to iC3b was $\sim 18$ fold higher than previously described for the isolated CR3 $\alpha_{M} I$ domain (30). We therefore investigated whether this was due to stronger binding through the $\alpha_{M} l$ domain in the context of the CR3 headpiece or whether CR3 contains one or more additional interaction sites for iC3b outside of the $\alpha_{M} I$ domain. For this purpose, we used the recombinant C3d fragment contained within C3dg but with the flexible remnants of the C3g fragment removed (30). As above, the apparent affinity of the CR3-C3d complex was measured using SPR (Fig. 2A-B). Because the binding kinetics were very fast and data could not be robustly fitted to a 1:1 interaction model, we instead performed a steady-state analysis and measured an apparent $K_{D}=515 \mathrm{nM}$ similar to the affinity of the $\alpha_{M} l$ domain for iC3b (30). This suggests that embedding of the $\alpha_{M} l$ into the CR3 headpiece does not significantly change its affinity for the C3d moiety in iC3b. Next, we performed an SPR-based competition assay where we measured the binding of the $\mathrm{CR} 3$ headpiece to immobilized iC3b in the presence of increasing concentrations of free C3b, iC3b and C3d (Fig. 2C-E). Fluid phase iC3b and C3d competed for binding to the immobilized iC3b whereas C3b did not, demonstrating that the competition was ligand-specific. Fluid phase iC3b robustly competed for CR3 binding whereas, by contrast, presence of C3d only produced a marginal decrease in the binding signal even at a 50-fold molar excess of C3d. In 
summary, our SPR data demonstrated that additional contacts, outside of the $\alpha_{M} l$ domain:TE interface described by X-ray crystallography (30), contribute to the CR3 interaction with iC3b.

\section{iC3b adopts an extended but stable conformation}

After $\mathrm{C} 3 \mathrm{~b}$ is cleaved by $\mathrm{FI}$ to form $\mathrm{iC} 3 \mathrm{~b}$, the CUB domain is thought to become disordered and the TE domain no longer closely associates with the MG-ring (2-5). To understand the structural state of the CUB and TE domains after iC3b formation, we recorded SAXS data on iC3b, and for comparison C3b (Fig. S2A). Guinier analysis did not suggest interparticle effects. C3b and iC3b exhibited $R_{g}$ values of $49 \AA$, and $53 \AA$ respectively (Fig. S2B-C), well in line with earlier reports (3, 38). Comparison of the Kratky plots (Supplementary Fig. S2A) showed that iC3b adopts a more extended structure than C3b. To investigate the solution conformation of ic3b further, we performed rigid body modeling against the scattering data of iC3b. We also performed the same analysis against the data for C3b as a control (Fig. S2D-F). The C3c fragment excluding the C345c domain was modelled as a single rigid body. The C345c domain was modelled independently due to the known flexibility of the domain, but was restrained to maintain the disulphide to MG7. For C3b the CUB and TE domains were treated as a single body, while for iC3b the TE domain was modelled as a rigid body and the cleaved CUB domain was modeled as connected dummy residues. For C3b all output models clustered closely, showing a slight detachment of the TE domain from the MG1 domain (Fig. S2E-F) consistent with prior results (38). For iC3b, 100 independent rigid body models were generated with two different initial positions of the TE domain. In the first initial model, the TE domain was positioned as in C3b, and in the other the TE domain was positioned next to the $\mathrm{C} 345 \mathrm{c}$ domain. Independent of the initial position of the TE domain, the 10 models with the best fit to the experimental data clustered closely in terms of conformation. In these models, the cleaved CUB domain adopts an extended conformation with the TE domain positioned far from MG1 and the rest of the MG-ring (Fig. 3A). Apart from being detached from the MG-ring, the CUB-TE moiety has also rotated so that the TE domain is located towards the MG4 domain edge of $\mathrm{C} 3 \mathrm{c}$, whereas in crystal structures of $\mathrm{C} 3 \mathrm{~b}$ the TE domain forms contacts with the MG1 domain (Fig. 3A). In contrast, other models with a significantly worse fit to the data had the 
TE domain close to the C345c domain. A comparison of these two classes of rigid body models reveals that even though they are very different with respect to the location of the TE domain, their mass distribution is similar (Fig. 3C-D). In summary, the degraded CUB domain adopts an extended conformation, leading to the TE domain detaching significantly from the MG core to a preferred position rather than being randomly located relative to the $\mathrm{C} 3 \mathrm{c}$ moiety.

\section{The iC3b-CR3 complex is compact}

To characterize the solution structure of the iC3b:CR3 headpiece complex we collected synchrotron inline SEC-SAXS data. The forward scattering elution profile displayed two peaks - the first one corresponding to the complex, and the second one corresponding to excess iC3b (Fig. 4A). The $\mathrm{R}_{\mathrm{g}}$ was stable throughout the first peak demonstrating that the CR3 remained saturated with iC3b during the SEC run in agreement with the $30 \mathrm{nM} \mathrm{K}_{\mathrm{D}}$ observed by SPR. A Guinier analysis of the scattering curve did not indicate interparticle effects and suggested a $R_{g}$ of $67 \AA$ for the CR3-iC3b complex (Fig S3A). Based on calculation of the pair distribution function the $D_{\max }$ for the complex was $\sim 260 \AA$ (Fig. S3C). In support of a well-defined and compact CR3:iC3b complex, this is only slightly larger than the $\sim 200 \AA$ we observe for the iC3b and the CR3 headpiece monomers.

To obtain models of the iC3b:CR3 complex and to identify the parts of iC3b involved in the complex with CR3 apart from the TE domain, we performed rigid body refinement against the SAXS data. Within the complex, iC3b was modelled as for free iC3b except that the TE domain was fixed relative to the $\alpha_{M} l$ domain of $C R 3$, to maintain the interaction known from the crystal structure. The resulting rigid body was connected through two distance restrains between the $\alpha_{M} l$ domain and the $\alpha_{M} \beta$-propeller. This allows the $\alpha_{M} I$ domain to rotate relative to the rest of the CR3 headpiece. The remaining fragment of the CR3 headpiece was modelled as a single rigid body in the open conformation. To improve the model fit to the data, the position of the $\mathrm{C} 3 \mathrm{c}$ fragment in the best initial solutions was used as a new initial model, and the translation and rotation of the rigid bodies were sampled more finely. After two iterations, this strategy converged and resulted in the best model fitting the data with $\chi^{2}=1.8$ (Fig. 4C). The 15 best fitting models with $1.8<\chi^{2}<2.2$ were 
classified into three tight clusters (Fig. 4D-F \& Fig. S3D-F). In all the analyzed models, iC3b adopts a conformation markedly different from that of unbound iC3b. Relocation of the C3c moiety brings the iC3b TE domain close to the MG-ring (Fig. 4D-F) as opposed to unbound iC3b where the TE domain is located distantly from the MG-ring (Fig. 3A). However, our models do not allow us to define a unique position of the $\mathrm{C} 3 \mathrm{c}$ fragment relative to the rest of the complex as rather different C3c orientations are observed in the three clusters of models (Fig. 4D-F \& Fig. S3D-F).

The DAMMIF program was used to generate 40 ab initio models from the CR3:iC3b scattering curve. The models were subsequently clustered using DAMCLUST, which gave one major cluster containing 32 models. The models were aligned and averaged, which resulted in a flat and extended ab initio envelope, with similar dimensions to the rigid body models (Fig. 4G). Taken together, our SAXS data support a model where upon CR3 recognition, the C3C and TE domain moieties of iC $3 \mathrm{~b}$ are brought closer to one another because of recognition by CR3 of regions outside the TE domain. This results in a compact receptor-ligand complex which upon extrapolation to an opsonized cell recognized by CR3 predicts the C3c moiety of iC3b to be overall much closer to the activator rather than extended away from the surface as predicted for C3b (Fig. $4 \mathrm{H}$ ).

\section{Discussion}

Prior crystal structure and biophysical experiments of the $\alpha_{M} l-C 3 d$ complex defined the core of the iC3b-CR3 interaction centered on the coordination of the divalent cation in the $\alpha_{M}$ I MIDAS by an aspartate from the iC3b TE domain. It was further demonstrated that C3d and C3dg bound the $\alpha_{\mathrm{M}}$ I-domain with an affinity resembling that of the iC3b- $\alpha_{M}$ I-domain interaction $(30,39)$. This $C 3 d(g)$ CR3 interaction appears to have physiological relevance since C3dg mediated erythrophagocytosis may occur in individuals suffering from paroxysmal nocturnal hemoglobinuria (39). However, other regions in CR3 beside the $\alpha_{M} \mathrm{I}$-domain must contribute to iC3b binding, since its deletion leaves residual iC3b affinity in CR3 (34). Both the $\alpha_{M} \beta$-propeller and the $\beta_{2}$ I-like domain have been implicated in interaction with iC $3 \mathrm{~b}(34,36,40,41)$, and on the iC $3 \mathrm{~b}$ side, mutations in the iC3b Nt$\alpha^{\prime}$ region associating with the MG7 domain weaken the iC3b-CR3 interaction (35). All these prior 
lines of evidence are consistent with a higher affinity of the CR3 headpiece for iC3b as compared to C3dg.

Prior studies have disagreed upon the affinity of CR3 for iC3b, and have been limited by a number of factors, including activation state of the receptor, purity of the protein preparations, and degree of oligomerization of the ic3b preparations $(31,32)$. We now quantitate the binding using highly pure and well-characterized proteins, and show that CR3 recognized iC3b with a $K_{D}$ of $30 \mathrm{nM}$ in $\mathrm{Mg}^{2+} / \mathrm{Ca}^{2+}$. This is 14-fold higher than the $515 \mathrm{nM}$ observed for the CR3-C3d interaction in $\mathrm{Mg}^{2+} / \mathrm{Ca}^{2+}$, and is to our knowledge, the highest monovalent affinity measured between $\mathrm{C} 3 \mathrm{~b}$, ic $3 \mathrm{~b}$, $\mathrm{C} 3 \mathrm{dg}$ and their five complement receptors. Although the difference in $\Delta \mathrm{G}$ for these two dissociation constants only corresponds to $7 \mathrm{~kJ} / \mathrm{mol}$, due to multiple complexes formed between a phagocyte and an iC3b opsonized activator, a strong effect is predicted to result in vivo. Hence, if our findings translate to cell-bound $\mathrm{CR} 3$, the much more efficient phagocytosis of iC3b tagged objects compared to those tagged by C3dg can be rationalized. Importantly, it is only the combination of the CR3 headpiece and iC3b that results in this high affinity interaction, as both the CR3-C3d (this study) and the $\alpha_{M}$ l-iC3b interactions (30) have $K_{D}$ values of 500-600 nM. Thus, omission of the CUB and C3c moities of iC3b, or of the $\beta I$ and $\beta$-propeller moieties of CR3, result in lower affinities, agreeing with our finding that these moieties come close to one another in iC3b-CR3 complexes.

Our rigid body models of the CR3:iC3b complex suggest that the C3c moiety of iC $3 b$ is in close proximity to CR3. A comparison with our models of unbound iC3b suggest that the TE domain is brought closer to C3C moiety upon CR3 binding. The heterogeneity in our SAXS models with respect to the location of the $\mathrm{C} 3 \mathrm{c}$ moiety of iC3b relative to $\mathrm{CR} 3$ may reflect the in vivo situation. Alternatively, the limited information content present in the underlying SAXS data can give rise to rigid body models that from a structural point of view are quite different but fit the data equally well. The system is also challenging for SAXS rigid body refinement due to the difficulty of modeling the remnants of the CUB domain. Our refinement strategy was also conservative using only four rigid bodies to describe a $300 \mathrm{kDa}$ complex with a $\mathrm{D}_{\max }$ of $26 \mathrm{~nm}$. Despite these limitations, our SAXS 
models are overall compatible with an ensemble of CR3-iC3b orientations seen in a recent nsEM study (2), except that those complexes showed more separation between the end of the C3c MG ring bearing the $\mathrm{C} 345 \mathrm{c}$ domain and the CR3 $\beta_{1}-\beta$-propeller interface. The authors suggested a second, three-dimensional contact formed between the integrin and ic $3 \mathrm{~b}$ that was less stable than the aMI-TE domain contact and was susceptible to disruption when iC3b-CR3 complexes adsorbed to the grid and become largely planar. These results are consistent with lack of co-planarity of the MG ring of $C 3 c$ and the integrin headpiece in our rigid body models. We have also been unable to observe compact iC3b-CR3 complex particles using nsEM grids despite extensive efforts and use of gradient fixation (42).

The structural arrangement of iC $3 b$ has been somewhat controversial, with prior SAXS and nsEM studies disagreeing both within and between methods $(2-4,43,44)$. In most EM-based studies, the TE domain was observed to be flexibly attached to the C3c moiety $(2-4,44)$. However, in one EM study the TE domain was found stably associated with the C345c domain (43). This study also presented SAXS data with significantly lower values of $D_{\max }$ and $R_{g}$ than those observed by us and others $(3,43)$. Our data are more in line with other EM-based models of iC3b, where the TE domain is loosely associated with the C3c moiety through an extended CUB domain $(2-4,44)$.

Inhibition of specific CR3-ligand interactions has been investigated for decades, but is complicated by the plethora of structurally diverse CR3 ligands reported. Multiple CR3 function blocking antibodies are known, e.g. (45-47) and small molecules known as leukadherins binding CR3 and suppressing outside-in signaling upon ligand binding reduce inflammation and suppress tumor growth in animal models of cancer $(48,49)$. Recent developments in neurobiology are likely to fuel the interest for an iC3b-specific CR3 inhibitor. During development, activation of the classical pathway of complement on weakly signaling synapses leads to iC $3 \mathrm{~b}$ deposition and recognition by CR3-expressing microglia, which phagocytize the iC3b opsonized synapses $(8,9,50)$. Very recently, microglia CR3 was shown to support complement-dependent synapse elimination by microglia as a mechanism underlying the forgetting of remote memories (51). However, the same 
pathway that ensures correct development and removal of remote memories by pruning excess synapses, is linked to Alzheimer's disease (13), frontotemporal dementia (FTD) (50) and spinal muscular atrophy (52). Our demonstration of a stable and compact complex between ic $3 \mathrm{~b}$ and the CR3 headpiece with a dissociation constant in the low nanomolar range offers hope for the development of molecules capable of specifically interfering with the iC3b:CR3 interaction while preserving the ability of CR3 to recognize its many other non-complement ligands.

\section{Materials and methods}

\section{Generation of a stable cell line expressing the CR3 headpiece fragment}

The coding sequence of the human $\mathrm{CR} 3 \alpha_{\mathrm{M}}$-chain residues 17-773 containing the glycan knockout mutations N225R/N680R and $\beta_{2}$-chain residues 23-504 were cloned into the pIRES2-EGFP based in-house vectors ET10c and ET10b respectively. The ET10c vector contains a Human Rhinovirus (HRV) 3C protease recognition site, an acid coiled-coil region, a Strepll-tag and a His 6 -tag on the 3', directly in-frame with the cloning site. The open reading frame was subsequently subcloned into pcDNA3.1(+). The ET10b vector contains an HRV 3C protease recognition site, a basic coiled-coil region, and a His 6 -tag directly in-frame with the cloning site. The CR3 $\alpha_{M}$ - and $\beta_{2}$-chain were cotransfected into human embryonic kidney (HEK) 2935 GnTi- cells (ATCC). The selection antibiotics Hygromycin B and $\mathrm{G} 418$ at $200 \mu \mathrm{g} / \mathrm{mL}$ and $1 \mathrm{~g} / \mathrm{mL}$, respectively, were added to the cultures 48 hours post transfection. After selection the cells were assessed for GFP expression using fluorescence-activated cell sorting, and the top $5 \%$ expressing clones were seeded in a 96 well cell culture plate. A final selection step was performed on the cell supernatants using sandwich ELISA by capturing the CR3 headpiece by use of an anti-CR3 $\alpha_{M}$-chain antibody (CBRM 1/2), and detected using a biotinylated anti-CR3 $\beta_{2}$-chain antibody (IB4).

\section{Expression and purification of CR3 headpiece}

The CR3 headpiece stably transfected HEK293S cells were kept as adhesion cell culture growing in Dulbecco's Modification of Eagle's Medium (DMEM) GlutaMAX (Gibco) supplemented with 10 \% (v/v) fetal bovine serum (FBS), 20 mM HEPES pH 7.5, 1 \% Penicillin-Streptomycin (Gibco), 200 
$\mu \mathrm{g} / \mathrm{mL}$ Hygromycin B (Sigma-Aldrich) and $200 \mu \mathrm{g} / \mathrm{mL}$ G418 (Sigma-Aldrich). Before large scale purify cation, the cells were adapted to serum-free medium. The cell supernatant was harvested by centrifugation and subsequently filtered through $0.2 \mu \mathrm{m}$ filters. The cleared cell supernatant was supplemented with $50 \mathrm{mM}$ TRIS pH 8, $500 \mathrm{mM} \mathrm{NaCl}, 5 \mathrm{mM} \mathrm{MgCl}_{2}$ and $1 \mathrm{mM} \mathrm{CaCl}_{2}$ and applied to a $5 \mathrm{~mL}$ HisTrap Excel (GE Healthcare). Afterwards the column was washed with $40 \mathrm{~mL}$ of 20 $\mathrm{mM}$ TRIS pH $8,1.5 \mathrm{M} \mathrm{NaCl}, 5 \mathrm{mM} \mathrm{MgCl}_{2}, 1 \mathrm{mM} \mathrm{CaCl}_{2}$ and the protein was eluted in $20 \mathrm{~mL}$ of 20 $\mathrm{mM}$ TRIS $\mathrm{pH}$ 8, $150 \mathrm{mM} \mathrm{NaCl}, 5 \mathrm{mM} \mathrm{MgCl}$, $1 \mathrm{mM} \mathrm{CaCl}$, $400 \mathrm{mM}$ imidazole. The elution was applied to a $1 \mathrm{~mL}$ StrepTactin column (GE Healthcare) equilibrated in $20 \mathrm{mM}$ HEPES pH 7.5, 150 $\mathrm{mM} \mathrm{NaCl}, 5 \mathrm{mM} \mathrm{MgCl}_{2}, 1 \mathrm{mM} \mathrm{CaCl}$. The column was washed in $20 \mathrm{mM}$ HEPES pH 7.5, $150 \mathrm{mM}$ $\mathrm{NaCl}, 5 \mathrm{mM} \mathrm{MgCl}_{2}, 1 \mathrm{mM} \mathrm{CaCl}_{2}$ and the protein was subsequently eluted in $20 \mathrm{mM}$ HEPES pH 7.5, $150 \mathrm{mM} \mathrm{NaCl}, 5 \mathrm{mM} \mathrm{MgCl}_{2}, 1 \mathrm{mM} \mathrm{CaCl}_{2}$, $2.5 \mathrm{mM}$ D-desthiobiotin. 3C rhinovirus protease was added in a 1:10 mass ratio to $\mathrm{CR} 3$ and the reaction was allowed to proceed at $4^{\circ} \mathrm{C}$ overnight. $\mathrm{A}$ final polishing step was performed by size exclusion chromatography (SEC) on a $24 \mathrm{~mL}$ Superdex 200 increase (GE Healthcare) equilibrated in $20 \mathrm{mM} \mathrm{HEPES} \mathrm{pH} \mathrm{7.5,} 150 \mathrm{mM} \mathrm{NaCl}, 5 \mathrm{mM} \mathrm{MgCl} 2$ and $1 \mathrm{mM} \mathrm{CaCl}_{2}$.

\section{Surface plasmon resonance assays}

Human C3d was expressed and purified as described in (30). C3b and iC3b was generated and purified as described in (37). The experiments were performed on a Biacore T200 instrument with a running buffer containing $20 \mathrm{mM}$ HEPES pH 7.5, $150 \mathrm{mM} \mathrm{NaCl}, 5 \mathrm{mM} \mathrm{MgCl}$, $1 \mathrm{mM} \mathrm{CaCl} 2$ unless otherwise stated. Streptavidin was immobilized on a CMD500M chip (XanTec Bioanalytics) to 200 response units. C3d, or iC3b biotinylated on the thioester cysteine was injected on the chip until the surface was saturated. For the kinetics experiments using iC3b, the CR3 headpiece was injected in a concentration series ranging from $0.3215 \mathrm{nM}$ to $100 \mathrm{nM}$, whereas for C3d, the CR3 headpiece was injected in a concentration series ranging from $3.25 \mathrm{nM}$ to $2000 \mathrm{nM}$. The surface was regenerated by using a buffer containing 50 mM EDTA, $1 \mathrm{M} \mathrm{NaCl}, 100 \mathrm{mM}$ HEPES pH 7.5. The data were analyzed using a 1:1 binding model, and the reported on- and off-rates are averages 
of three independent experiments. The kinetic experiment with ic3b on the surface was repeated three times in the buffer containing $20 \mathrm{mM}$ HEPES pH 7.5, $150 \mathrm{mM} \mathrm{NaCl}, 1 \mathrm{mM} \mathrm{MnCl}$, $0.2 \mathrm{mM}$ $\mathrm{CaCl}_{2}$ as well. The competition assays were performed on the ic $3 \mathrm{~b}$ surface where $20 \mathrm{mM}$ of CR3 headpiece was injected either alone, or pre-incubated on ice for 1 hour with 10, 20, 50, 100, 200, or $1000 \mathrm{nM}$ of iC3b, C3d or C3b respectively. All experiments were performed in triplicates.

\section{Analytical SEC analysis}

For analyzing the effect of different divalent cations on the oligomeric state of $\mathrm{CR} 3,50 \mu \mathrm{L}$ of $\mathrm{CR} 3$ headpiece at $2 \mu \mathrm{g} / \mu \mathrm{L}$ was diluted four-fold in either $20 \mathrm{mM}$ HEPES pH 7.5, $150 \mathrm{mM} \mathrm{NaCl}, 1 \mathrm{mM}$ $\mathrm{MnCl}_{2}, 0.2 \mathrm{mM} \mathrm{CaCl}_{2} ; 20 \mathrm{mM}$ HEPES pH 7.5, $150 \mathrm{mM} \mathrm{NaCl}, 5 \mathrm{mM} \mathrm{MgCl}_{2}, 1 \mathrm{mM} \mathrm{CaCl}$; or $20 \mathrm{mM}$ HEPES pH 7.5, $150 \mathrm{mM} \mathrm{NaCl}, 5 \mathrm{mM} \mathrm{NiCl}_{2}, 1 \mathrm{mM} \mathrm{CaCl}_{2}$. The protein was incubated for 1 hour at room temperature before being injected on a $24 \mathrm{~mL}$ Superdex 200 increase equilibrated in the respective protein dilution buffer. For analyzing the complex formation between CR3 and iC3b, 15 $\mu \mathrm{g}$ of iC3b was mixed with 1.1 fold molar excess of CR3. The sample was injected on a $2.4 \mathrm{~mL}$ Superdex 200 increase equilibrated in 20 mM HEPES pH 7.5, $150 \mathrm{mM} \mathrm{NaCl}, 5 \mathrm{mM} \mathrm{MgCl}$, $1 \mathrm{mM}$ $\mathrm{CaCl}_{2}$. Control experiments injecting either $\mathrm{CR} 3$ or ic3b in the same amount was also performed.

\section{SAXS data collection and analysis}

In-line SEC-SAXS data for the CR3 headpiece in complex with iC3b were collected at the P12 beamline at PETRA III, Hamburg, Germany (53). Scattering was recorded from the elution of a 24 $\mathrm{mL}$ Superdex 200 increase equilibrated in $20 \mathrm{mM}$ HEPES pH 7.5, $150 \mathrm{mM} \mathrm{NaCl}, 5 \mathrm{mM} \mathrm{MgCl}$, 1 $\mathrm{mM} \mathrm{CaCl} 2$ with a flow rate of $0.25 \mathrm{~mL} / \mathrm{min}$. The CR3 headpiece was mixed with 1.2 fold molar excess of iC3b and subsequently injected on the SEC column. Each frame during the SEC-SAXS run covers a $0.955 \mathrm{~s}$ exposure performed every second. The sample-to-detector distance was 3.0 m covering $0.02<q<4.8 \mathrm{~nm}^{-1}\left(q=4 \pi \cdot \sin \theta \cdot \lambda^{-1}\right.$, where $2 \theta$ is the scattering angle). Normalization and radial averaging was performed at the beamline using the automated pipeline $(53,54)$. Buffer subtraction was performed using CHROMIXS (55). Due to the high level of averaging performed 
during SEC-SAXS data processing, detector imperfections most likely led to the error estimates being underestimated at low $\mathrm{q}$, as revealed an indirect Fourier transform of the data. The error estimates were therefore rescaled by Err=Err original $\cdot\left(1+2.5 \cdot \exp \left(-7 q^{2}\right)\right)$. Forty ab initio models were generated using dammif in slow-mode, and the resulting models were clustered using damclust (56). This lead to six clusters with only one major cluster containing 32 of the 40 models. The models of each cluster was subsequently averaged and filtered yielding the final models of each cluster. SAXS measurements of CR3 headpiece, C3b and iC3b in batch mode, were likewise collected at the P12 beamline. The data were collected in a temperature-controlled capillary at $20^{\circ} \mathrm{C}$ using a PILATUS 2M pixel detector (DECTRIS) with $\lambda=1.240 \AA$. The sample-to-detector distance was $3.0 \mathrm{~m}$ covering $0.02<q<4.8 \mathrm{~nm}^{-1}$. Samples of CR3 were prepared at $0.7,1.3,1.7$ and 2.9 $\mathrm{mg} / \mathrm{mL}$, and samples of $\mathrm{C} 3 \mathrm{~b}$, and iC3b were prepared at 7.3 , and $13.0 \mathrm{mg} / \mathrm{mL}$ respectively, where after data was collected with twenty exposures of $45 \mathrm{~ms}$. Radial averaging, buffer subtraction and concentration scaling was performed by the automated beamline pipeline (57) and the pair distribution function was calculated by indirect Fourier transformation using GNOM (58).

\section{SAXS rigid body modelling}

Rigid body modelling using CORAL (56), was performed for C3b, iC3b and the CR3:iC3b complex against their corresponding SAXS curves. Input domains for C3b and iC3b was extracted from RCSB entry 6EHG (37), after aligning the principal inertial vectors of the structure with the Cartesian axes using alpraxin (59). For C3b the MG1-6, MG7, C345c and the CUB-TE fragment were extracted as separate files. The rigid bodies were connected using LINK statements to model the missing linker regions. The MG1-7 and MG8 rigid bodies were fixed relative to each other such that the MG ring was kept intact, and a distance restraint of $6 \AA$ was applied between Cys873 in the MG8 domain and Cys1513 in the C345c domain to maintain their disulphide bridge. Twenty rigid body models were generated with CORAL, which are all shown in fig. S2E. For iC3b a similar scheme was used, except that the CUB domain was modelled using dummy residues to represent all three flexible stretches of the degraded domain. This was done 
by (1) introducing a LINK statement connecting the MG7 C-terminal residue to the $\mathrm{N}$-terminal residue of the TE domain. (2) Introducing a CTER statement at the C-terminal residue of the TE domain. (3) Introducing a NTER statement at the N-terminal residue of the MG8 domain. One hundred coral runs were performed, half using an input model where the TE domain was placed in a C3b like conformation and the other half where the TE domain was placed beside the C345c domain. No systematic differences could be observed between the models coming from either input models. The ten models with the best fit to the data are shown in Fig. 3A. For the CR3:iC3b complex iC3b was modelled as for free iC3b, except that the TE domain was fixed relative to the $\alpha_{M} I$ domain of CR3, to keep the interface known from the crystal structure of the complex (RCSB entry $4 M 76(60)$ ) intact. The $N$ - and C-terminal residues of the CR3 $\alpha_{M} l$ was restrained with two distance restraints of either 7 or $9 \AA$ to the $\alpha_{M} \beta$-propeller since with distance restraints of $5 \AA$ very few models obtained $\chi^{2}<3$. The rest of the CR3 headpiece was kept as a single rigid body, using a homology model based on $\alpha \times \beta_{2}(4 \mathrm{NEH})$, modelled in the open conformation using the $\beta$ chain from the crystal structure of $\alpha_{\| 1} \beta_{3}(R C S B$ entry 2VDR (61)). Three consecutive rounds of rigid body modelling were performed, in rounds 2 and 3 starting from the best fitting model of the prior round. To sample the rigid body movement more finely, the CORAL parameters "angular step" and "spatial step" were decreased from the default $20^{\circ}$ and $5 \AA$ to $10^{\circ}$ and $2.5 \AA$ respectively during optimization. No systematic differences could be observed between the models coming from different input models and subject to 7 or $9 \AA$ distance restraints. Hence, a total of 135 output models were analyzed and the best 15 models having $1.8<\chi^{2}<2.2$ were analyzed.

\section{Acknowledgements and funding sources}

We thank the staff at the P12 beamline at PETRAIII for help during data collection, and Lise Arleth and Jan Skov Pedersen for discussions on correction of SAXS data. The authors would like to acknowledge Christine Schar for assistance with SPR and Karen Margrethe Nielsen for technical 
support. This work was supported by the Lundbeck Foundation (BRAINSTRUC, grant no. R1552015-2666) and the Danish Foundation for Independent Research (grant no 4181-00137).

Conflict of interest. The authors declare no conflicts of interest in relation to this manuscript. 


\section{References}

1. Zipfel, P. F., and C. Skerka. 2009. Complement regulators and inhibitory proteins. Nat Rev Immunol 9: 729-740.

2. Xu, S., J. Wang, J. H. Wang, and T. A. Springer. 2017. Distinct recognition of complement iC3b by integrins alphaXbeta2 and alphaMbeta2. Proc Natl Acad SciU S A 114: 3403-3408.

3. Xue, X. G., J. Wu, D. Ricklin, F. Forneris, P. Di Crescenzio, C. Q. Schmidt, J. Granneman, T. H. Sharp, J. D. Lambris, and P. Gros. 2017. Regulator-dependent mechanisms of C3b processing by factor I allow differentiation of immune responses. Nat Struct Mol Biol 24: 643-+.

4. Nishida, N., T. Walz, and T. A. Springer. 2006. Structural transitions of complement component C3 and its activation products. Proc Natl Acad Sci U S A 103: 19737-19742.

5. Papanastasiou, M., S. Koutsogiannaki, Y. Sarigiannis, B. V. Geisbrecht, D. Ricklin, and J. D. Lambris. 2017. Structural Implications for the Formation and Function of the Complement Effector Protein iC3b. J Immunol 198: 3326-3335.

6. Vorup-Jensen, T., and R. K. Jensen. 2018. Structural Immunology of Complement Receptors 3 and 4. Front Immunol 9: 2716.

7. Erdei, A., S. Lukacsi, B. Macsik-Valent, Z. Nagy-Balo, I. Kurucz, and Z. Bajtay. 2019. Nonidentical twins: Different faces of CR3 and CR4 in myeloid and lymphoid cells of mice and men. Semin Cell Dev Biol 85: 110-121.

8. Stevens, B., N. J. Allen, L. E. Vazquez, G. R. Howell, K. S. Christopherson, N. Nouri, K. D. Micheva, A. K. Mehalow, A. D. Huberman, B. Stafford, A. Sher, A. M. Litke, J. D. Lambris, S. J. Smith, S. W. John, and B. A. Barres. 2007. The classical complement cascade mediates CNS synapse elimination. Cell 131: 1164-1178.

9. Schafer, D. P., E. K. Lehrman, A. G. Kautzman, R. Koyama, A. R. Mardinly, R. Yamasaki, R. M. Ransohoff, M. E. Greenberg, B. A. Barres, and B. Stevens. 2012. Microglia sculpt postnatal neural circuits in an activity and complement-dependent manner. Neuron 74: 691-705.

10. Wakselman, S., C. Bechade, A. Roumier, D. Bernard, A. Triller, and A. Bessis. 2008. Developmental neuronal death in hippocampus requires the microglial CD11b integrin and DAP12 immunoreceptor. J Neurosci 28: 8138-8143.

11. Jiang, L., S. H. Chen, C. H. Chu, S. J. Wang, E. Oyarzabal, B. Wilson, V. Sanders, K. Xie, Q. Wang, and J. S. Hong. 2015. A novel role of microglial NADPH oxidase in mediating extrasynaptic function of norepinephrine in regulating brain immune homeostasis. Glia 63: 1057-1072.

12. Kadioglu, A., K. De Filippo, M. Bangert, V. E. Fernandes, L. Richards, K. Jones, P. W. Andrew, and N. Hogg. 2011. The integrins Mac-1 and alpha4beta1 perform crucial roles in neutrophil and T cell recruitment to lungs during Streptococcus pneumoniae infection. J Immunol 186: 5907-5915.

13. Hong, S., V. F. Beja-Glasser, B. M. Nfonoyim, A. Frouin, S. Li, S. Ramakrishnan, K. M. Merry, Q. Shi, A. Rosenthal, B. A. Barres, C. A. Lemere, D. J. Selkoe, and B. Stevens. 2016. Complement and microglia mediate early synapse loss in Alzheimer mouse models. Science 352: 712-716.

14. Heesters, B. A., C. E. van der Poel, A. Das, and M. C. Carroll. 2016. Antigen Presentation to B Cells. Trends Immunol 37: 844-854. 
15. Li, J., and T. A. Springer. 2018. Energy landscape differences among integrins establish the framework for understanding activation. J Cell Biol 217: 397-412.

16. Luo, B. H., and T. A. Springer. 2006. Integrin structures and conformational signaling. Curr Opin Cell Biol 18: 579-586.

17. Springer, T. A., and M. L. Dustin. 2012. Integrin inside-out signaling and the immunological synapse. Curr Opin Cell Biol 24: 107-115.

18. Li, J., and T. A. Springer. 2017. Integrin extension enables ultrasensitive regulation by cytoskeletal force. Proc Natl Acad Sci U S A 114: 4685-4690.

19. Diamond, M. S., J. Garcia-Aguilar, J. K. Bickford, A. L. Corbi, and T. A. Springer. 1993. The I domain is a major recognition site on the leukocyte integrin Mac-1 (CD11b/CD18) for four distinct adhesion ligands. J Cell Biol 120: 1031-1043.

20. Chavakis, T., A. Bierhaus, N. Al-Fakhri, D. Schneider, S. Witte, T. Linn, M. Nagashima, J. Morser, B. Arnold, K. T. Preissner, and P. P. Nawroth. 2003. The pattern recognition receptor (RAGE) is a counterreceptor for leukocyte integrins: a novel pathway for inflammatory cell recruitment. J Exp Med 198: 1507-1515.

21. Lishko, V. K., V. P. Yakubenko, T. P. Ugarova, and N. P. Podolnikova. 2018. Leukocyte integrin Mac-1 (CD11b/CD18, alphaMbeta2, CR3) acts as a functional receptor for platelet factor 4. J Biol Chem 293: 6869-6882.

22. Liu, Y. S., L. F. Wang, X. S. Cheng, Y. N. Huo, X. M. Ouyang, L. Y. Liang, Y. Lin, J. F. Wu, J. L. Ren, and B. Guleng. 2019. The pattern-recognition molecule mindin binds integrin Mac-1 to promote macrophage phagocytosis via Syk activation and NF-kappaB p65 translocation. J Cell Mol Med 23: 3402-3416.

23. Morgan, J., M. Saleem, R. Ng, C. Armstrong, S. S. Wong, S. G. Caulton, A. Fickling, H. E. L. Williams, A. D. Munday, J. A. Lopez, M. S. Searle, and J. Emsley. 2019. Structural basis of the leukocyte integrin Mac-1 I-domain interactions with the platelet glycoprotein $\mathrm{lb}$. Blood Adv 3: 1450-1459.

24. Saggu, G., K. Okubo, Y. Chen, R. Vattepu, N. Tsuboi, F. Rosetti, X. Cullere, N. Washburn, S. Tahir, A. M. Rosado, S. M. Holland, R. M. Anthony, M. Sen, C. Zhu, and T. N. Mayadas. 2018. Cis interaction between sialylated FcgammaRIIA and the alphal-domain of Mac-1 limits antibody-mediated neutrophil recruitment. Nat Commun 9: 5058.

25. Wolf, D., J. D. Hohmann, A. Wiedemann, K. Bledzka, H. Blankenbach, T. Marchini, K. Gutte, K. Zeschky, N. Bassler, N. Hoppe, A. O. Rodriguez, N. Herr, I. Hilgendorf, P. Stachon, F. Willecke, D. Duerschmied, C. von zur Muhlen, D. A. Soloviev, L. Zhang, C. Bode, E. F. Plow, P. Libby, K. Peter, and A. Zirlik. 2011. Binding of CD40L to Mac-1's I-domain involves the EQLKKSKTL motif and mediates leukocyte recruitment and atherosclerosis--but does not affect immunity and thrombosis in mice. Circ Res 109: 1269-1279.

26. Zhang, X., G. Bajic, G. R. Andersen, S. H. Christiansen, and T. Vorup-Jensen. 2016. The cationic peptide LL-37 binds Mac-1 (CD11b/CD18) with a low dissociation rate and promotes phagocytosis. Biochim Biophys Acta 1864: 471-478.

27. DuMont, A. L., P. Yoong, C. J. Day, F. Alonzo, 3rd, W. H. McDonald, M. P. Jennings, and V. J. Torres. 2013. Staphylococcus aureus LukAB cytotoxin kills human neutrophils by targeting the CD11b subunit of the integrin Mac-1. Proc Natl Acad Sci U S A 110: 1079410799.

28. Ranganathan, S., C. Cao, J. Catania, M. Migliorini, L. Zhang, and D. K. Strickland. 2011. Molecular basis for the interaction of low density lipoprotein receptor-related protein 1 
(LRP1) with integrin alphaMbeta2: identification of binding sites within alphaMbeta2 for LRP1. J Biol Chem 286: 30535-30541.

29. Vorup-Jensen, T. 2012. On the roles of polyvalent binding in immune recognition: perspectives in the nanoscience of immunology and the immune response to nanomedicines. Adv Drug Deliv Rev 64: 1759-1781.

30. Bajic, G., L. Yatime, R. B. Sim, T. Vorup-Jensen, and G. R. Andersen. 2013. Structural insight on the recognition of surface-bound opsonins by the integrin I domain of complement receptor 3. Proc Natl Acad Sci U S A 110: 16426-16431.

31. Cai, T. Q., and S. D. Wright. 1995. Energetics of leukocyte integrin activation. J Biol Chem 270: 14358-14365.

32. Gordon, D. L., G. M. Johnson, and M. K. Hostetter. 1987. Characteristics of iC3b binding to human polymorphonuclear leucocytes. Immunology 60: 553-558.

33. Gaither, T. A., I. Vargas, S. Inada, and M. M. Frank. 1987. The complement fragment C3d facilitates phagocytosis by monocytes. Immunology 62: 405-411.

34. Yalamanchili, P., C. Lu, C. Oxvig, and T. A. Springer. 2000. Folding and function of I domaindeleted Mac-1 and lymphocyte function-associated antigen-1. J Biol Chem 275: 2187721882.

35. Taniguchi-Sidle, A., and D. E. Isenman. 1994. Interactions of human complement component C3 with factor $B$ and with complement receptors type 1 (CR1, CD35) and type 3 (CR3, CD11b/CD18) involve an acidic sequence at the N-terminus of C3 alpha'-chain. $J$ Immunol 153: 5285-5302.

36. Li, Y., and L. Zhang. 2003. The fourth blade within the beta-propeller is involved specifically in C3bi recognition by integrin alpha M beta 2. J Biol Chem 278: 34395-34402.

37. Jensen, R. K., R. Pihl, T. A. F. Gadeberg, J. K. Jensen, K. R. Andersen, S. Thiel, N. S. Laursen, and G. R. Andersen. 2018. A potent complement factor C3-specific nanobody inhibiting multiple functions in the alternative pathway of human and murine complement. $J$ Biol Chem 293: 6269-6281.

38. Rodriguez, E., R. Nan, K. Y. Li, J. Gor, and S. J. Perkins. 2015. A Revised Mechanism for the Activation of Complement C3 to C3b A MOLECULAR EXPLANATION OF A DISEASEASSOCIATED POLYMORPHISM. Journal of Biological Chemistry 290: 2334-2350.

39. Lin, Z., C. Q. Schmidt, S. Koutsogiannaki, P. Ricci, A. M. Risitano, J. D. Lambris, and D. Ricklin. 2015. Complement C3dg-mediated erythrophagocytosis: implications for paroxysmal nocturnal hemoglobinuria. Blood 126: 891-894.

40. MacPherson, M., H. S. Lek, A. Prescott, and S. C. Fagerholm. 2011. A systemic lupus erythematosus-associated $\mathrm{R} 77 \mathrm{H}$ substitution in the $\mathrm{CD} 11 \mathrm{~b}$ chain of the Mac-1 integrin compromises leukocyte adhesion and phagocytosis. The Journal of biological chemistry 286: 17303-17310.

41. Xiong, Y.-M., T. a. Haas, and L. Zhang. 2002. Identification of functional segments within the beta2I-domain of integrin alphaMbeta2. The Journal of biological chemistry 277: 46639-46644.

42. Stark, H. 2010. GraFix: stabilization of fragile macromolecular complexes for single particle cryo-EM. Methods Enzymol 481: 109-126.

43. Alcorlo, M., R. Martinez-Barricarte, F. J. Fernandez, C. Rodriguez-Gallego, A. Round, M. C. Vega, C. L. Harris, S. R. de Cordoba, and O. Llorca. 2011. Unique structure of iC3b resolved at a resolution of 24 A by 3D-electron microscopy. Proc Natl Acad Sci U S A 108: 1323613240. 
44. Chen, X., Y. Yu, L. Z. Mi, T. Walz, and T. A. Springer. 2012. Molecular basis for complement recognition by integrin alphaXbeta2. Proc Natl Acad Sci U S A 109: 4586-4591.

45. Oxvig, C., C. Lu, and T. A. Springer. 1999. Conformational changes in tertiary structure near the ligand binding site of an integrin I domain. Proc Natl Acad Sci U S A 96: 22152220.

46. Mahalingam, B., K. Ajroud, J. L. Alonso, S. Anand, B. D. Adair, A. L. Horenstein, F. Malavasi, J. P. Xiong, and M. A. Arnaout. 2011. Stable coordination of the inhibitory Ca2+ ion at the metal ion-dependent adhesion site in integrin CD11b/CD18 by an antibody-derived ligand aspartate: implications for integrin regulation and structure-based drug design. J Immunol 187: 6393-6401.

47. Wolf, D., N. Anto-Michel, H. Blankenbach, A. Wiedemann, K. Buscher, J. D. Hohmann, B. Lim, M. Bauml, A. Marki, M. Mauler, D. Duerschmied, Z. Fan, H. Winkels, D. Sidler, P. Diehl, D. M. Zajonc, I. Hilgendorf, P. Stachon, T. Marchini, F. Willecke, M. Schell, B. Sommer, C. von Zur Muhlen, J. Reinohl, T. Gerhardt, E. F. Plow, V. Yakubenko, P. Libby, C. Bode, K. Ley, K. Peter, and A. Zirlik. 2018. A ligand-specific blockade of the integrin Mac-1 selectively targets pathologic inflammation while maintaining protective host-defense. Nat Commun 9: 525.

48. Schmid, M. C., S. Q. Khan, M. M. Kaneda, P. Pathria, R. Shepard, T. L. Louis, S. Anand, G. Woo, C. Leem, M. H. Faridi, T. Geraghty, A. Rajagopalan, S. Gupta, M. Ahmed, R. I. Vazquez-Padron, D. A. Cheresh, V. Gupta, and J. A. Varner. 2018. Integrin CD11b activation drives anti-tumor innate immunity. Nat Commun 9: 5379.

49. Maiguel, D., M. H. Faridi, C. Wei, Y. Kuwano, K. M. Balla, D. Hernandez, C. J. Barth, G. Lugo, M. Donnelly, A. Nayer, L. F. Moita, S. Schurer, D. Traver, P. Ruiz, R. I. Vazquez-Padron, K. Ley, J. Reiser, and V. Gupta. 2011. Small molecule-mediated activation of the integrin CD11b/CD18 reduces inflammatory disease. Sci Signal 4: ra57.

50. Lui, H., J. Zhang, S. R. Makinson, M. K. Cahill, K. W. Kelley, H. Y. Huang, Y. Shang, M. C. Oldham, L. H. Martens, F. Gao, G. Coppola, S. A. Sloan, C. L. Hsieh, C. C. Kim, E. H. Bigio, S. Weintraub, M. M. Mesulam, R. Rademakers, I. R. Mackenzie, W. W. Seeley, A. Karydas, B. L. Miller, B. Borroni, R. Ghidoni, R. V. Farese, Jr., J. T. Paz, B. A. Barres, and E. J. Huang. 2016. Progranulin Deficiency Promotes Circuit-Specific Synaptic Pruning by Microglia via Complement Activation. Cell 165: 921-935.

51. Wang, C., H. Yue, Z. Hu, Y. Shen, J. Ma, J. Li, X. D. Wang, L. Wang, B. Sun, P. Shi, L. Wang, and Y. Gu. 2020. Microglia mediate forgetting via complement-dependent synaptic elimination. Science 367: 688-694.

52. Vukojicic, A., N. Delestree, E. V. Fletcher, J. G. Pagiazitis, S. Sankaranarayanan, T. A. Yednock, B. A. Barres, and G. Z. Mentis. 2019. The Classical Complement Pathway Mediates Microglia-Dependent Remodeling of Spinal Motor Circuits during Development and in SMA. Cell Rep 29: 3087-3100 e3087.

53. Blanchet, C. E., A. Spilotros, F. Schwemmer, M. A. Graewert, A. Kikhney, C. M. Jeffries, D. Franke, D. Mark, R. Zengerle, F. Cipriani, S. Fiedler, M. Roessle, and D. I. Svergun. 2015. Versatile sample environments and automation for biological solution X-ray scattering experiments at the P12 beamline (PETRA III, DESY). J Appl Crystallogr 48: 431-443.

54. Graewert, M. A., D. Franke, C. M. Jeffries, C. E. Blanchet, D. Ruskule, K. Kuhle, A. Flieger, B. Schafer, B. Tartsch, R. Meijers, and D. I. Svergun. 2015. Automated pipeline for purification, biophysical and x-ray analysis of biomacromolecular solutions. Sci Rep 5: 10734. 
55. Panjkovich, A., and D. I. Svergun. 2018. CHROMIXS: automatic and interactive analysis of chromatography-coupled small-angle X-ray scattering data. Bioinformatics 34: 19441946.

56. Petoukhov, M. V., D. Franke, A. V. Shkumatov, G. Tria, A. G. Kikhney, M. Gajda, C. Gorba, H. D. Mertens, P. V. Konarev, and D. I. Svergun. 2012. New developments in the ATSAS program package for small-angle scattering data analysis. J App/ Crystallogr 45: 342-350.

57. Franke, D., A. G. Kikhney, and D. I. Svergun. 2012. Automated acquisition and analysis of small angle X-ray scattering data. Nucl Instrum Meth A 689: 52-59.

58. Svergun, D. I. 1992. Determination of the Regularization Parameter in Indirect-Transform Methods Using Perceptual Criteria. Journal of Applied Crystallography 25: 495-503.

59. Kozin, M. B., and D. I. Svergun. 2001. Automated matching of high- and low-resolution structural models. Journal of Applied Crystallography 34: 33-41.

60. Bajic, G., L. Yatime, R. B. Sim, T. Vorup-Jensen, and G. R. Andersen. 2013. Structural insight on the recognition of surface-bound opsonins by the integrin I domain of complement receptor 3. P Natl Acad Sci USA 110: 16426-16431.

61. Xiao, T., J. Takagi, B. S. Coller, J. H. Wang, and T. A. Springer. 2004. Structural basis for allostery in integrins and binding to fibrinogen-mimetic therapeutics. Nature 432: 59-67. 


\section{Figures}

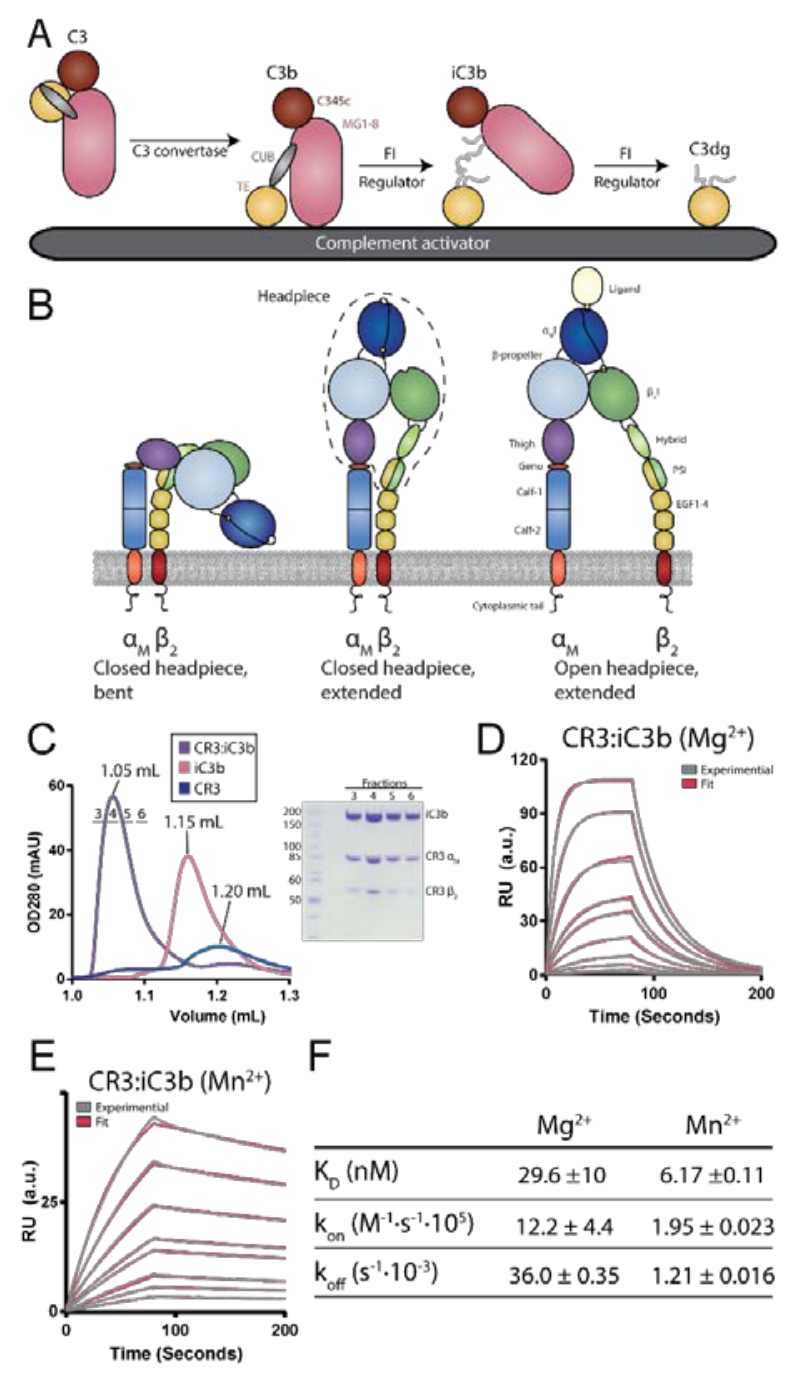

Figure 1. Characterization of the CR3 headpiece fragment. C3 is cleaved by a C3 convertase into the opsonin $\mathrm{C} 3 \mathrm{~b}$, which is deposited on the activator surface. $\mathrm{C} 3 \mathrm{~b}$ can then be further degraded into iC3b by the $\mathrm{FI}$ protease, aided by a regulator binding to $\mathrm{C} 3 \mathrm{~b}$. FI may further degrade iC $3 \mathrm{~b}$ into C3dg. B. CR3 adopts three different overall conformations. The bent conformation (left) and intermediate, extended closed conformation (middle) are low affinity. The extended, open conformation is $\sim 1,000$-fold higher affinity (right). The dashed line indicates the headpiece fragment. C. SEC analysis of the complex formation between CR3 and iC3b. The complex elutes significantly earlier compared to both $\mathrm{iC} 3 \mathrm{~b}$ and $\mathrm{CR} 3$ alone. The fractions indicated in the chromatogram by a horizontal line is analysed by non-reducing SDS-PAGE. D. SPR sensorgrams for the interaction of CR3, injected on an iC3b surface in a $\mathrm{Mg}^{2+}$ buffer. CR3 was injected at 100, $50,25,12.5,10,5,2.5,1.25,0.625,0.3125 \mathrm{nM}$. The raw curves are shown in grey and the fit are shown in red. The dissociation constant calculated as $\mathrm{K}_{\mathrm{D}}=\mathrm{k}_{\text {off }} / \mathrm{K}_{\text {on }}$ is indicated. The on- and off-rates are the average of three independent experiments. $\mathrm{E}$. As in panel $\mathrm{D}$, but in a $\mathrm{Mn}^{2+}$ buffer, only curves for CR3 concentrations 25, 12.5, 10, 5, 2.5, 1.25, 0.625. $0.3125 \mathrm{nM}$ are displayed. F. Table summarizing the kinetic constants determined by SPR, \pm the standard deviation. 

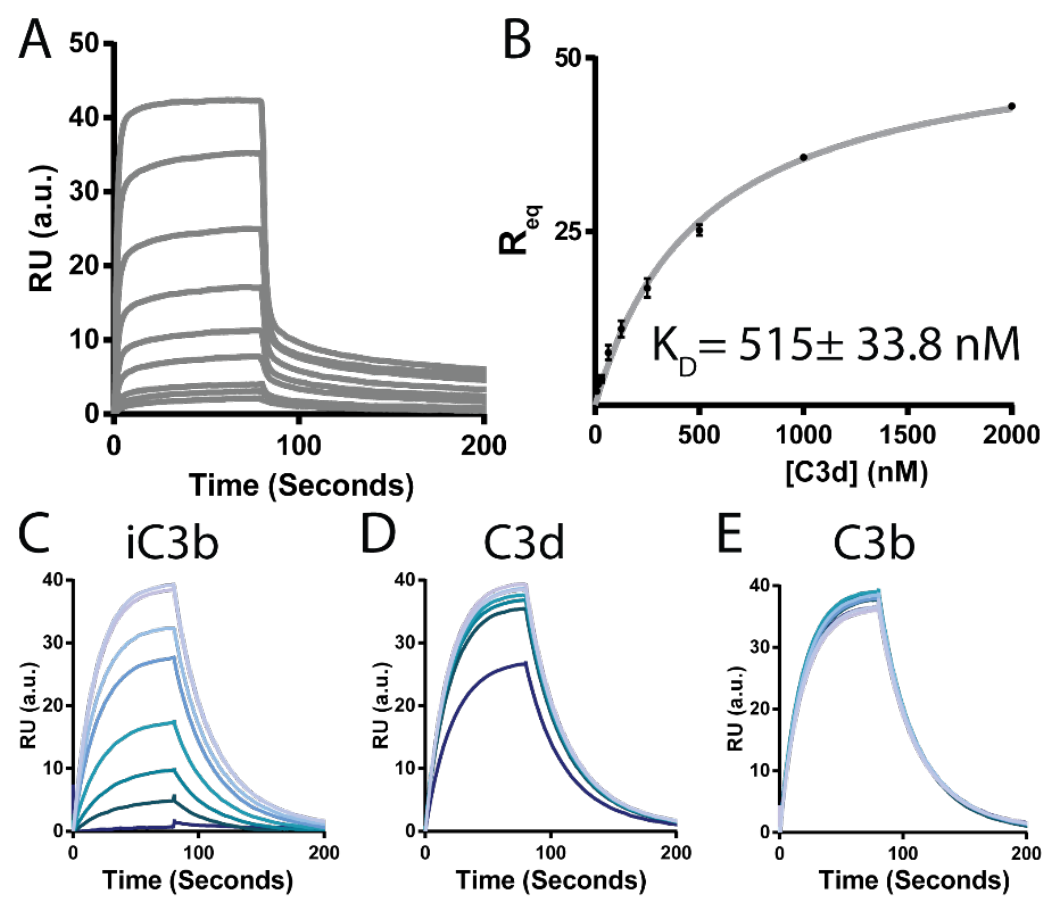

E $\quad \mathrm{C} 3 \mathrm{~b}$

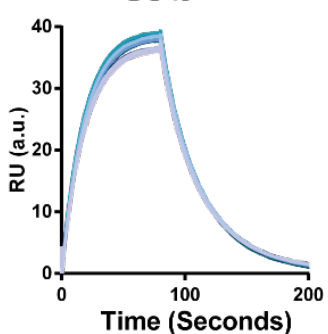

$\begin{array}{cccc}0 \mathrm{nM} & 10 \mathrm{nM} & \square 0 \mathrm{nM} & \square 200 \mathrm{mM} \\ 0 \mathrm{nM} & \mathbf{2 0} \mathrm{nM} & \square 100 \mathrm{nM} & \square 1000 \mathrm{mM}\end{array}$

Figure 2. Analysis of the interaction between C3d and CR3. A. Sensorgrams from an SPR experiment where the CR3 headpiece at $2000,1000,500,250,125,62.5,31.25,15.63,7.81 \mathrm{nM}$ was injected on a C3d coated sensor in a $\mathrm{Mg}^{2+}$ buffer. B. Steady-state analysis of SPR experiments in panel A. Average values \pm the standard deviation for three repetitions are plotted, and the $K_{D}$ value is determined by non-linear regression and given \pm the standard deviation. The resulting $K_{D}$ value is 17 fold higher than the $K_{D}$ determined for the CR3:iC3b complex. C-E. Sensorgrams of SPR competition assays where $20 \mathrm{nM}$ of CR3 was incubated with variable concentrations of iC3b (C), C3d (D), or C3b (E) before being injected on an iC3b-coated sensor. 


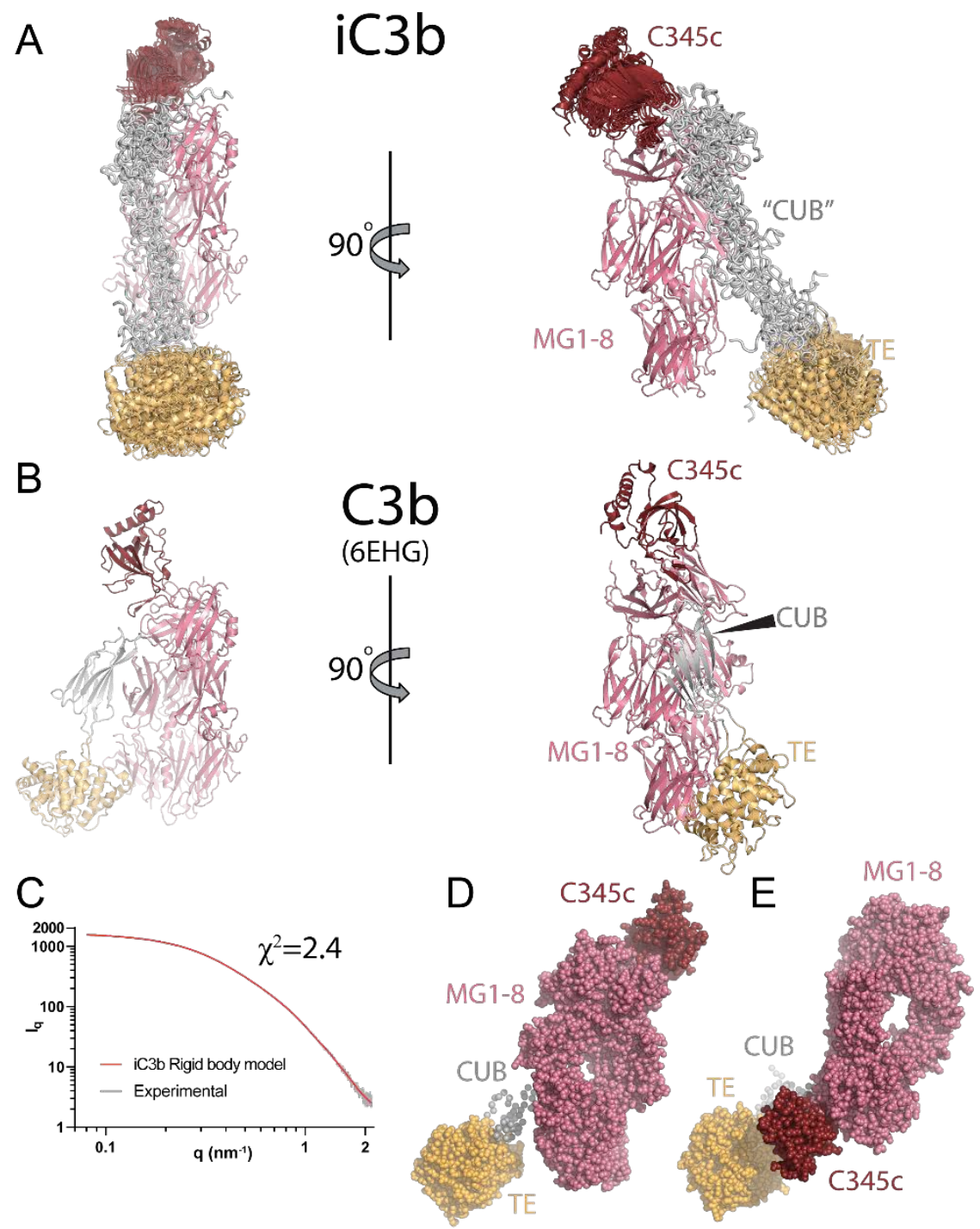

Figure 3. SAXS rigid body analysis of iC3b. A. The 10 rigid body models with the lowest $\chi^{2}-$ value aligned on the MG1-6 domains. The models cluster closely together with their thioester domain displaced from the MG-ring, connected by an extended degraded CUB domain. C3b is presented in the same orientation for comparison. B. Experimental scattering curve (grey) compared with the curve calculated (red) from the ic3b rigid model shown in C. C. The ic3b model with the lowest $\chi^{2}$-value shown in a spheres representation. D. An alternative iC $3 \mathrm{~b}$ rigid body model where the thioester domain is in close proximity to the $\mathrm{C} 345 \mathrm{c}$ domain. Notice how the mass distribution resembles that of the best fitting model in panel $\mathrm{C}$. 

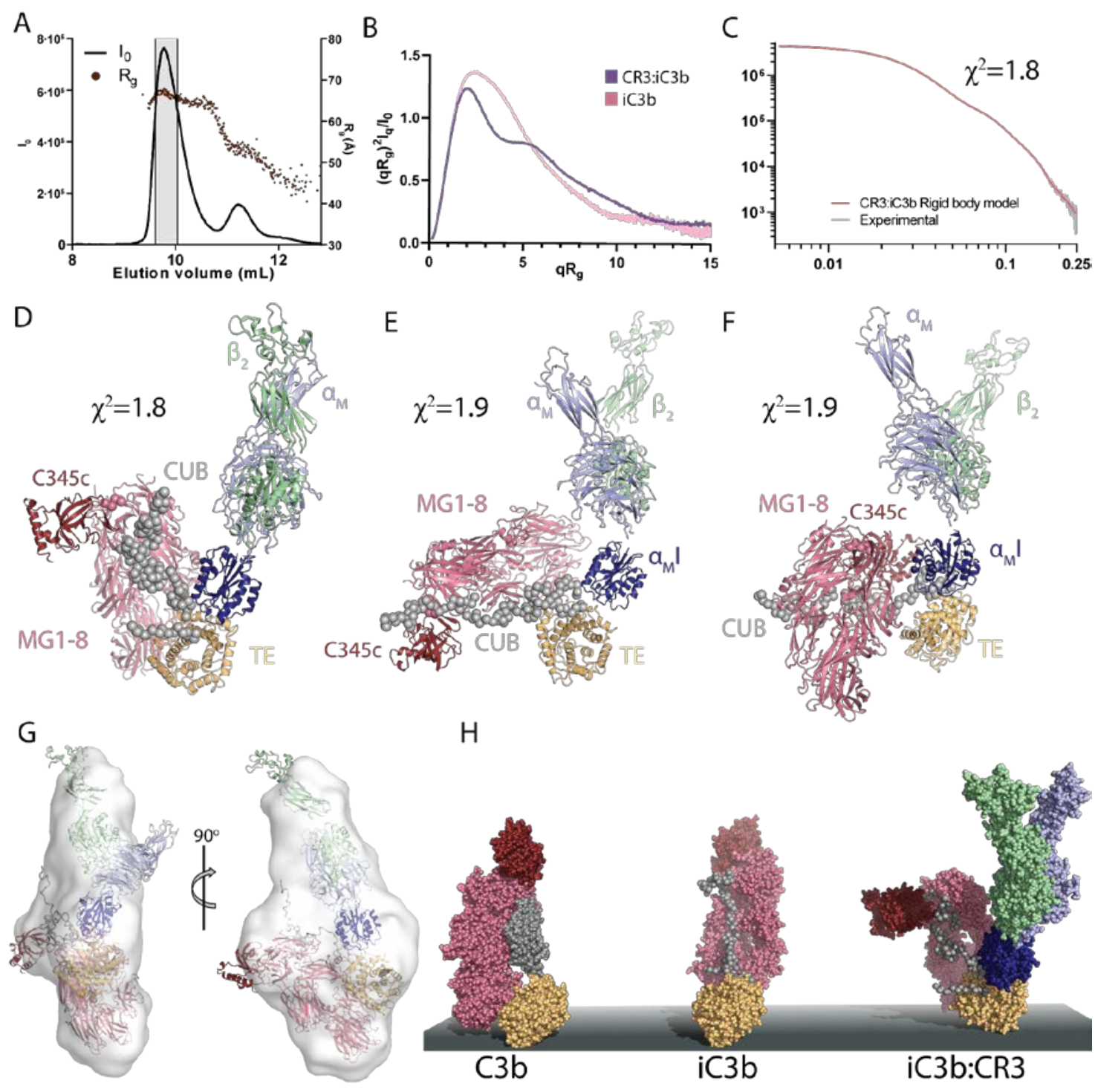

$\mathrm{H}$
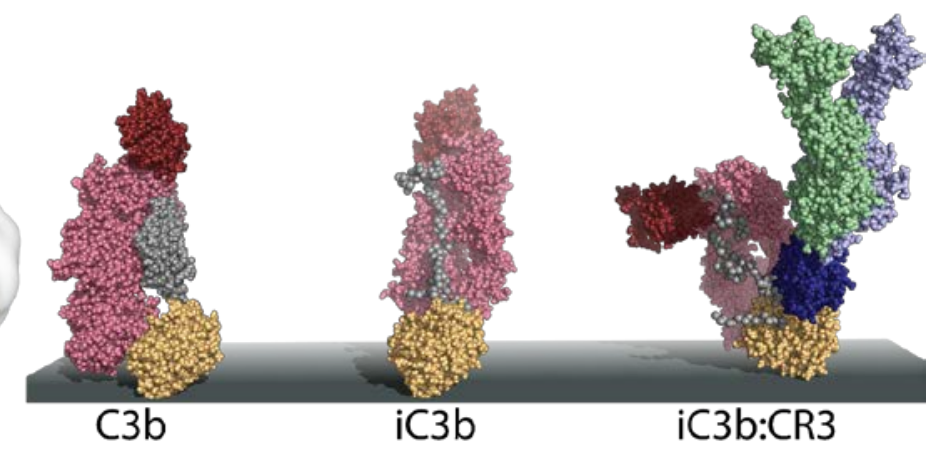

Figure 4. SEC-SAXS analysis of the iC3b:CR3 in complex. A. The forward scattering and $\mathrm{R}_{\mathrm{g}}$ of each frame during the SEC-SAXS experiment of the CR3:iC3b complex plotted as a function of the elution volume. The two peaks contain the CR3:iC3b complex and excess free iC3b, respectively. The experimental scattering curve for the CR3:iC3b complex was obtained from the shaded area. B. The Guiner-normalized Kratky plot of the CR3:iC3b complex (purple) and iC3b alone (pink). C. Experimental scattering curve (grey) compared with the curve calculated (red) from the CR3:iC3b rigid model shown in panel D. D-F. Representative rigid body models of the three clusters of rigid body models. Upon CR3 recognition, the CUB remnants reorganizes, leading to a more compact iC3b conformation, but the exact position of $\mathrm{C} 3 \mathrm{c}$ varies significantly between the three clusters. G. Average model of $32 \mathrm{ab}$ initio models calculated from the CR3:iC3b SEC-SAXS data aligned to the rigid body model of the complex in panel D. H. Models of C3b, iC3b and the CR3:iC3b complex on an activator surface with coloring as in panels D-F. $\mathrm{C} 3 \mathrm{~b}$ is deposited on the surface and cleaved by FI into iC3b. The degraded CUB domain in iC3b extends releasing the $\mathrm{C} 3 \mathrm{c}$ moiety of iC $3 \mathrm{~b}$ from the TE domain. Upon CR3 recognition, the CUB domain reorganizes substantially. 